\title{
Las creencias de la docente de educación física sobre la obesidad en la niñez de edad escolar
}

\section{Beliefs of Physical Education Female Teachers about Obesity in School-age Children}

\author{
Hilda Patricia Núñez-Rivas? \\ Instituto Costarricense de Investigación y \\ Enseñanza en Nutrición y Salud (INCIENSA) \\ Cartago, Costa Rica \\ hnunez@inciensa.sa.cr \\ Natalia Campos-Saborío ${ }^{2}$ \\ Vicerrectoría de Investigación \\ Universidad Estatal a Distancia \\ San José, Costa Rica \\ ncampos@uned.ac.cr \\ Ileana Holst-Schumacher ${ }^{3}$ \\ Facultad de Microbiología \\ Universidad de Costa Rica \\ San José, Costa Rica \\ ileana.holst@ucr.ac.cr \\ Flory Virginia Alfaro-Mora ${ }^{4}$ \\ Instituto Costarricense de Investigación y \\ Enseñanza en Nutrición y Salud (INCIENSA) \\ Cartago, Costa Ria \\ valfaro@inciensa.sa.cr
}

Recibido 08 de febrero de 2013 • Corregido 10 de marzo de 2013 • Aceptado 13 de marzo de 2013

1 Doctora en Educación, Instituto Costarricense de Investigación y Enseñanza en Nutrición y Salud (INCIENSA). Nutricionista-investigadora, Unidad de Salud y Nutrición, Costa Rica.

2 Doctora en Educación, investigadora, Vicerrectoría de Investigación, Universidad Estatal a Distancia (UNED), Costa Rica.

3 Máster en Química Clínica, Facultad de Microbiología, Universidad de Costa Rica, catedrática-investigadora del Centro de Investigación en Hematología y Trastornos Afines (CIHATA), Costa Rica.

4 Licenciada en Trabajo Social, Instituto Costarricense de Investigación y Enseñanza en Nutrición y Salud (INCIENSA). Trabajadora Social-investigadora, Unidad de Salud y Nutrición, Costa Rica. 
URL: http://www.una.ac.cr/educare

CORREO: educare@una.cr

Resumen. El propósito de este artículo es comprender cómo las creencias del personal docente de Educación Física (EF) acerca de la obesidad de niños y niñas en edad escolar, favorecen u obstaculizan la prevención de esta condición. Para tal fin se empleó el método etnográfico en una escuela urbana pública. Las técnicas de recolección de datos fueron: observación participante, entrevistas a profundidad, grupos focales y análisis documental. Estas permitieron recoger datos abundantes y ricos de significados, que al ser triangulados por fuentes le dieron validez a los resultados de la investigación. Hallazgos informan sobre la actividad física limitada al ámbito áulico, la obesidad como condición que dificulta la participación de los niños y niñas en EF, la exclusión de niños con obesidad en la práctica de esta asignatura, el privilegio que da la docente a los estudiantes con menos dificultad en la actividad física y su desatención a los que requieren más apoyo, y cómo sus estudiantes modelan sus creencias.

Palabras claves. Creencias, obesidad, Educación física, Revista Electrónica Educare.

Abstract. The purpose of this paper is to understand how the beliefs of Physical Education (PE) teachers about obesity in school-age children may help or hinder the prevention efforts on that area. For this study, we applied the ethnographic method in an urban public school. Data was collected through the following techniques: observation of participants, in-depth interviews, focal groups, and documentary analysis. Rich and abundant data was collected and triangulated using sources that validated the research results. The findings report on physical activity limited to the classroom environment, obesity as a barrier for children to participate in $\mathrm{PE}$, exclusion of children with obesity from PE practices, female teachers providing privileges to students who have fewer limitations for physical activity and neglecting those who need encouragement, and how students shape their own beliefs.

Keywords. Physical Education, teachers, beliefs, child obesity, theoretical-practical foundation.

La infancia es una etapa decisiva de la vida humana; durante esta se adquieren conocimientos y se aprenden comportamientos en los diversos entornos, los que a su vez, configuran las creencias, las actitudes y las expectativas de conductas futuras. En relación con la educación para la salud, las creencias del personal docente son de suma importancia, porque ellas, conjuntamente con las de los padres y madres de familia y las de la comunidad, forman parte en la definición de actitudes y comportamientos de los niños y las niñas, en un aspecto tan preciado para el ser humano como la salud.

Las creencias del personal docente constituyen verdaderas teorías implícitas sobre el aprendizaje y la enseñanza, profundamente enraizadas, no solo en la cultura escolar dominante, sino también en las actividades de enseñanza cotidianas (el planeamiento, la organización del aula, la práctica educativa, la evaluación, entre otros). Las creencias, además, forman parte de la propia estructura cognitiva del profesorado y del alumnado (Pozo, 2000). Ellas están insertas en las ideas, percepciones y actitudes de docentes, de manera consciente o inconsciente, y guían sus decisiones al planificar y poner en práctica su oferta académica (Durán, 2001).

Las creencias del personal docente de educación física (EF, en adelante) sobre la obesidad pueden favorecer u obstaculizar la formación de hábitos en la actividad, ejercicio físico y deporte de los niños y las niñas, factor importante en la prevención de la obesidad.

6

Hilda Patricia Núñez-Rivas, Natalia Campos-Saborío, lleana Holst-Schumacher y Flory Virginia Alfaro-Mora 
Por consiguiente, es pertinente conocer las creencias docentes sobre la obesidad en niños escolares, con el fin de conocerlas, aclararlas y someterlas a un proceso de cambio, si estas son perjudiciales a la educación de la salud del estudiantado.

En el presente estudio, se analiza cómo las creencias de la docente de EF, de una escuela urbana de San José, Costa Rica, benefician o dificultan la formación de hábitos para la prevención de la obesidad en la niñez escolar.

Ello, porque la obesidad es una patología prevalente en la niñez de países desarrollados y en vías de desarrollo. En Costa Rica, se reportó $34,5 \%$ de sobrepeso y $26,2 \%$ de obesidad, en una muestra representativa de 1,718 estudiantes de 7 a 12 años de edad (Núñez-Rivas, Monge-Rojas, León y Roselló, 2003). De acuerdo con estadísticas de las últimas encuestas nacionales de nutrición del 2008 al 2009, la obesidad en personas adultas costarricenses ha ido aumentando. En el caso de las mujeres, en 1982, 43\% tenía obesidad y sobrepeso; en 1996, un 46\% y en 2009 un 60\%. Referente a los hombres adultos de 1,2 millones, 754,342 tienen sobrepeso y obesidad (Díaz, 2012).

En consecuencia, es urgente analizar la problemática de la obesidad en la niñez, al estudiar las creencias de la docente de EF, sobre esta enfermedad, pues la actividad, el ejercicio físico y el deporte juegan un papel relevante en su prevención.

\section{Referente teórico}

El enfoque teórico-práctico de la EF ofrece el para qué, el porqué, el qué, el cómo, el dónde, el cuándo y el quienes realizaran los procesos de enseñanza y aprendizaje de la asignatura. De acuerdo con Hernández (1996), citado por (Moreno, 2010), hay cuatro tendencias actuales de la EF en el currículo escolar, a saber:

- Deportes: (...) corriente a partir de la cual se hace un traslado, casi mecánico, de los principios y procedimientos de la lógica del deporte de élite a la estructura de la educación física escolar, [donde] la competición deportiva y la victoria (...) [son] el centro de las prácticas escolares para buscar el prestigio institucional y personal.

- Condición física: la condición física también ha intentado convertirse en el centro hegemónico a partir del cual gire la educación física escolar. Según el autor en el que nos estamos centrando, los antecedentes de esta corriente habría que situarlos en la lógica científica cuya intencionalidad ha estado en crear las bases anatómicas y fisiológicas del movimiento humano. [Desde] esta corriente, es posible visualizar una asociación entre "(...) la condición física y una idea reduccionista de la salud, en cuanto a su concepción puramente física y objetiva" (Hernández, 1996, p. 60). 
URL: http://www.una.ac.cr/educare

CORREO: educare@una.cr

- Psicomotricidad: es bajo esta corriente donde comienza a pensarse en un cuerpo menos reduccionista que se aleja de lo puramente físico y objetivo. Es el cuerpo pensante el centro de esta tendencia, [al entender] que a través del movimiento corporal es posible el desarrollo intelectual de la persona. Si bien comienza a haber un alejamiento del conocido 'cuerpo máquina' como sustento de la educación física escolar y representado, principalmente, a partir de las dos concepciones anteriores, también es cierto que esta corriente estuvo focalizada en las edades más tempranas de la niñez, por lo que su fuerza en la educación física en general ha sido y es limitada.

- Expresión corporal: se orienta, principalmente, hacia el desarrollo de las capacidades rítmico expresivas del cuerpo que se expresan a través del movimiento. La creatividad natural en el ser humano el centro de su existencia, [por tanto argumenta] que los movimientos estereotipados presentes en el deporte de competición no [la] permiten y [la] legitiman (...). (p. 91)

Por otro lado, Vázquez (2001) examina formas de entender la asignatura, a partir de las diferentes concepciones del movimiento humano y las clasifica en: biomotriz, psicomotriz y expresiva. Estas se fundamentan en tres formas diferentes de concebir el cuerpo humano:

- Cuerpo máquina: objetivación del cuerpo a partir de la metáfora que permitiría entender que este se comporta como una máquina perfecta de la cual es posible predecir su comportamiento.

- Cuerpo psicosomático: relación entre cuerpo y mente como dos realidades que funcionan a partir de la información que ambas se transmiten.

- Cuerpo expresivo: el cuerpo como sistema de comunicación.

Al respecto, cabe resaltar el aporte de Portela (2001) sobre la creencia griega acerca del cuerpo humano, como una obra de arte (cuerpo perfecto, síntesis de lo bueno y lo bello, conectado con lo mental, lo moral y lo físico, que pugna por un desarrollo interior a partir del cultivo de la apariencia externa). Esta creencia se aproxima a la existente en la era del capitalismo, donde el cuerpo se concibe como objeto físico, concepción ubicada en una tendencia biologista y pragmática del ser humano, la cual define al cuerpo "(...) como simple objeto físico sometido a las leyes naturales, cognoscible como cualquier elemento biológico a quien se pueda controlar y sobre quien se predica y condiciona para evitar perturbaciones sociales" (párr. 19). Se hace referencia, entonces, a un cuerpo sometido, dominado y controlado por un sistema social que le dicta cómo debe ser. 
Desde esta perspectiva reduccionista [del cuerpo], se limitan los valores, capacidad y la potencialidad corporal. Se orienta [sic] y estructura [sic] las conductas esperadas y deseadas con el propósito de conservar la hegemonía sobre el cuerpo, y [con esta visión, la] educación [descarta] la construcción de un [ser humano] crítico y pensante. Denis (1980) lo [conceptualiza] así: "(...) abordar el cuerpo enseñado no supone pretender describir una realidad institucional, sino que se trata de determinar una realidad institucionalizada", esto se se cumple al ser sometido [el cuerpo] a un proceso, en donde, se le educa para insertarse en el contexto que lo reclama. Bernard (1985) lo clarifica aún más al mencionar que "... toda nuestra educación es en cierta medida... el ajuste de nuestro cuerpo de conformidad con las exigencias normativas de la sociedad". (Espinal, 2006, Del cuerpo a la corporeidad, párr. 6)

En la actualidad, la EF tiende a centrarse en el desarrollo físico del cuerpo y en el deporte, principalmente en el fútbol. Algunas tendencias relacionadas con los enfoques de EF tienen como eje el deporte y la condición física, con una concepción de cuerpo como objeto, sometido a la tecnología, la producción, el consumo, la publicidad (Bernard, 1985); por el contrario, el enfoque contemporáneo pretende ser holístico: es un cuerpo en situación y en acción, que muestra lo que soy, en dónde se integra lo físico, lo psíquico, lo espiritual, tomando en cuenta el contexto cultural en dónde vivimos (Moreno, 2010).

Las creencias del personal docente de esta asignatura tienen insumo de los enfoques de EF que adoptan en sus prácticas-, así como de sus constructos personales, elaborados de sus propias creencias y de aquellas del colectivo de la cultura en que se desenvuelven. De acuerdo con Rosales, Vicente y Fleitas (2005), es importante conocer las creencias de este personal docente, pues se reflejan en su enfoque sobre EF y en las creencias acerca de la didáctica de la asignatura. Docentes, graduados de la Universidad de Olguin, Cuba indican en orden de prioridad, las siguientes:

1er. lugar: Aprovechamiento del tiempo de clase, [sic] Definición correcta de los objetivos y tareas. Utilización adecuada de medios y métodos. Calidad de las áreas e implementos.

2do. lugar: El control y la organización de las clases.

3er. lugar: Alto rendimiento motor y oportunidad de práctica para todos.

4to. lugar: Correspondencia con las características de rendimiento de los estudiantes.

5to. lugar: Una buena motivación por la actividad. (Rosales et al., Preferencias, expectativas y creencias de los profesores, párr. 2-6). 
URL: http://www.una.ac.cr/educare

CORREO: educare@una.cr

De esta visión de la educación física, se desprende que las funciones de los docentes son:

Cumplir los objetivos de la clase, controlar y organizar, demostrar y explicar las tareas y motivar (...), hacer consciente los objetivos a lograr, (...) evaluar y ofrecer feedback y (...) la corrección de errores. (Rosales et al., 2005, Preferencias, expectativas y crrencias de los profesores, párr. 7)

En relación con los contenidos de los programas, estos se centran en el "(...) desarrollo de las habilidades motrices deportivas, la condición física, ocupando un segundo plano, los conocimientos teóricos y el desarrollo de características psicológicas de la personalidad, limitando la evaluación (...) a las dos primeras de las áreas mencionadas" (Rosales et al., 2005, Preferencias, expectativas y crrencias de los profesores, párr. 8). Estas funciones se identifican con un enfoque curricular rígido, de control, basado en la motricidad deportiva, la condición física, y dejan de lado lo psicosocial. Este enfoque concuerda con estudios realizados por (Allison, Pissanos, Turner y Law, 2000), cuyos resultados apuntan a que los docentes de EF priorizan el desarrollo de la habilidad motriz.

Según el estudio de Rosales et al. (2005), con estudiantes de la práctica educativa de EF, se percibe la relación profesor-estudiante en los siguientes términos:

- Necesidad de sentirse aceptado por el profesor y sus compañeros, y evitación del fracaso o el ridículo, sobre todo en el sexo femenino.

- Sentimientos de frustración por no tener un físico adecuado y no ser incluido en muchas ocasiones en las actividades de juego o competencia, siendo este tiempo para ellos, de inactividad

- Necesidad de ser valorado justamente, no solo por su físico y su rendimiento, en tiempo o centímetros, sino también por su esfuerzo y su participación en las clases.

- Deseos de elegir las actividades a realizar de acuerdo a sus características y preferencias.

- El desconocimiento de los beneficios que la actividad física le puede aportar en su formación como profesional, su estabilidad psíquica e incluso en su calidad de vida

- La necesidad de diversión en las clases y aceptarla como una actividad agradable.

- El miedo a hacer el ridículo y la necesidad de saber exactamente el por qué se hacen determinadas actividades. Lo que está relacionado con la participación y motivación. (El diario de vida, párr. 8-13)

Estas características de la relación docente-estudiante reflejan una práctica educativa anclada en el enfoque de condición física y de desarrollo de la parte motriz del ser humano, en 
detrimento de una visión fundamentada en la acción humana (Habermas1999) y en los saberes del individuo (Morin, 2003). Toro (2007, p. 24), citado por Moreno (2010, p. 96), define esta práctica así:

Este modelo se sustenta en la acción humana y no en el movimiento. Desde este enfoque se entiende al ser humano como un ser que configura mundos desde su accionar, plegado y pleno de sentido, de significados y símbolos, orientado hacia [Enseñanza de la Educación Física en] fines y afectos, ilusión e imaginación, proyección y potencia de sí mismo en u [sic] contexto comunitario, nunca aislado, pero al mismo tiempo irrenunciablemente convocado a decidir su propia existencia y la de los demás cercanos a él o ella, siempre más o menos consciente de quién es, con quienes está y dónde está.

El enfoque, mencionado por Toro, de carácter holístico, de praxis humana, participativo, creativo y contextualizado; se opone a los enfoques rígidos, individualistas, hedonistas y repetitivos, los cuales conciben el cuerpo humano como una máquina en movimiento, y no como un creador que comunica interacción humana, recreativa, y promotora de salud física, mental y espiritual.

Una de las características de la práctica docente citada se refiere a: "Necesidad de sentirse, aceptado por el profesor y sus compañeros, y evitación del fracaso o el ridículo, sobre todo en el sexo femenino" (Rosales et al., El diario de vida, párr. 8). Esta declaración evoca una necesidad del ser humano, sumamente importante: Sentirse aceptado. Van Breemen (1974, p. 1) apunta que "(...) ser aceptado quiere decir que las personas con quienes vivo me hacen sentir que realmente valgo y soy digno de respeto (...). Ser aceptado significa que me permitan ser como soy (...). No tengo, pues, que pasar por alguien que no soy!".

Así que en la EF, en general, y en cuanto a su relación con el tratamiento del género, hay mucho trabajo que hacer. Estudios relacionados con la educación física y el género señalan que: Hay docentes que creen que las diferencias entre estudiantes mujeres y estudiantes hombres en la práctica de la Educación Física son "insalvables" y otros que indican que son "salvables" (Trueba, 2008).

Aquellos que piensan las diferencias de género como insalvables expresan:

(...) la profesora de Hockey refiriéndose a que las mujeres "son todas unos quesos" y que no pueden hacer con los varones las clases mixtas, o como la profesora " $D$ " que estaba indignada porque le daban horas de educación física de un grupo femenino a un profesor varón, que según ella no es capaz de comprender "ciertas cosas" (por su condición de varón), también "C" cuando dijo que en Polimodal no se puede comparar a los varones y mujeres porque los varones son otra cosa (en referencia a que son superiores) (...). (Trueba, Profesorado, párr. 2) 
URL: http://www.una.ac.cr/educare

CORREO: educare@una.cr

Los docentes que creen que las diferencias de género son salvables dicen que: “(...) intentan destacar lo más objetivamente posible lo positivo y lo negativo de los grupos que les tocan. A pesar, incluso, de los prejuicios previos [de otros docentes]" (Trueba, Profesorado, párr. 3)

Otros docentes, que no le dan importancia a las diferencias de género en sus clases piensan que: "[Permitir el] vocabulario soez y despectivo hacia el género femenino o hacen bromas con sus alumnos sobre el tema (...)" es una condición normal (Trueba, Profesorado, párr. 4).

En general, todos estos docentes con creencias diferentes perciben que el objetivo principal de la EF es el desarrollo de las capacidades condicionales (fuerza, resistencia y velocidad) y otros objetivos relacionados con la integración, la recreación, la construcción de la ciudadanía, la educación por la libertad los consideran de menor importancia. Esto manifiesta que los enfoques de EF llevados a la práctica se aferran a los enfoques de deporte y condición física, como se planteó anteriormente.

Lo anterior se relaciona con los resultados de Cremades, Álvarez, Urruzola, Jaramillo y Hernández (1995), quienes estudiaron el tiempo libre en el marco de la coeducación. Destacan que los estereotipos de género tienen implicaciones en el desarrollo de las capacidades de las niñas, los niños y jóvenes, dado que a las mujeres y a los varones se les atribuyen, socioculturalmente, características y cualidades particulares, según su sexo, lo cual define actividades diferenciadas por género. Según esta construcción social, los niños realizan actividades físico-recreativas y lúdicas; las niñas, aquellas que son de tipo cultural, artísticas y manuales, entre otras (ver tabla 1).

Tabla 1

Desarrollo de capacidades en el tiempo libre en la infancia y la juventud

\begin{tabular}{ll}
\hline $\begin{array}{l}\text { Actividades físico-recreativas, lúdicas y } \\
\text { en la naturaleza }\end{array}$ & $\begin{array}{l}\text { Actividades culturales-artísticas, manuales prácticas y } \\
\text { sociales }\end{array}$ \\
\hline Fuerza & Destreza \\
Potencia & Cooperación \\
Resistencia & Cuidado \\
Competitividad & Respeto \\
Control del espacio & Creatividad \\
Autonomía & Expresión \\
Riesgo & Comunicación \\
Dominación & Habilidad \\
Agresividad & Resolución \\
\hline Atribuidas socioculturalmente a los varones & Atribuidas socioculturalmente a las niñas \\
\hline
\end{tabular}

Nota: Tomado de Cremades et al. (1995, p. 168) 
Referente a las creencias docentes, en relación con la obesidad de niños escolares, el estudio de Rashmi and Sushma Jaswal (2012), realizado en la India, señala que padres y madres de familia y docentes creen que la obesidad se debe a factores biológicos (trastornos biológicos en sus organismos) o psicológicos (la falta de afecto y de atención de parte de los padres y madres de familia, así como de los docentes, los cuales causan vacíos en los niños que estos llenan con la comida). Sin embargo, hay estudios, entre ellos el de Story, Neumark-Sztainer y French (2002), que demuestran que la obesidad se debe, en gran medida, a los estilos de vida: En primer lugar, los informantes padres y madres de familia y docentes consideran que los niños y niñas desarrollan adicción a la comida por la accesibilidad y preferencia por comidas específicas, y por sus creencias acerca de la preparación y manera de servirlas. En segundo lugar, el no hacer ejercicio y hábitos pobres de alimentación han contribuido a la obesidad.

En el estudio de Davis y Carpenter (2009) los resultados indican que la falta de actividad y ejercicio físico de los niños es una causa importante en la condición de obesidad, pues aquellos que no se involucran en la actividad física de manera sistemática, tienen un gran riesgo de convertirse en obesos.

El sedentarismo es uno de los factores de riesgo más reconocidos para la obesidad y otras enfermedades crónicas (Council on School Health, 2006; Council on Sports Medicine and Fitness; DeMattia, Lemont y Meurer, 2007; Floriani y Kennedy, 2007; Rennie, Wells, McCaffrey y Livingstone, 2006; Stice, Shaw y Marti, 2006). Incluso algunos estudios plantean que las clases de EF son una de las estrategias claves para combatir, desde edades tempranas, el sedentarismo y sus consecuencias (Kaur, Hyder y Poston, 2003; Singh et al., 2006).

Los resultados de estos estudios indican que la solución del sobrepeso y de la obesidad no reside en el tratamiento clínico, sino que requiere de programas educativos que concienticen a los padres y madres de familia y a los docentes de los riesgos en salud que tiene el sobrepeso y la obesidad en los niños escolares, para que realicen cambios en los hábitos de alimentación y de actividad y ejercicio físico.

Obrian et al. (2007), en el estudio sobre percepciones de futuros docentes de EF hacia los niños obesos, indican que estos docentes creen que los niños obesos son más lentos, perezosos, y menos inteligentes que los niños con peso normal. Los investigadores concluyen que la formación de docentes de EF necesita incluir programas para reducir percepciones negativas explícitas o implícitas acerca de la niñez con obesidad.

El estudio de Greenleaf y Weiller (2005) encuentra que las percepciones de los docentes de EF, sobre sus expectativas de niños con peso normal y niños obesos, referente a la fuerza, la flexibilidad, la resistencia, y la preparación física para coordinación y deporte de competencia son significativamente menores para los niños obesos que para los niños con peso normal. El estudio concluye que las bajas expectativas de los docentes acerca de la habilidad física de los niños obesos influye en el ofrecimiento de una oferta inclusiva en los programas de EF. 
URL: http://www.una.ac.cr/educare

CORREO: educare@una.cr

Los resultados del estudio de Núñez-Rivas et al. (2003) muestran cómo los niños y niñas escolares se apropian de las creencias y percepciones expresadas en estudios anteriores. En este estudio, se conocen patrones de comportamiento social entre las niñas y los niños; entre las niñas con obesidad y sus pares y entre los varones con obesidad y sus pares. Sus participaciones definieron, en diversas actividades y sobre todo en la práctica del fútbol y otros deportes de "competencia" los patrones mostrados en la tabla 2.

Tabla 2

Características atribuidas socioculturalmente a la población estudiantil, de acuerdo con su sexo y estado nutricional, que definen su participación en deportes y otras actividades

\begin{tabular}{llll}
\hline Niños & Niñas & Con obesidad & Sin obesidad \\
\hline - Groseros & • Delicadas & - Lentitud & • Rapidez/Velocidad \\
- Bruscos & - No les gusta ensuciarse & - Cansancio & • Agilidad \\
- Mandones/Dominantes & - Son malas para el fútbol & - Pereza & • Resistencia para \\
- Leales & - No corren & - Nula o poca movilidad & permanecer en \\
- Buenos amigos & - Buenas amigas & - Dificultad para correr & actividad \\
- Se defienden entre ellos & - Creídas & - Nula o poca resistencia & • Competitividad \\
- Son buenos para el & - Vanidosas & para permanecer en & \\
fútbol & - Comparten & actividad & \\
& & - Si es varón, puede ser & \\
& & portero & \\
\hline
\end{tabular}

Nota: Esta tabla es tomada de Núñez-Rivas (2007).

Las áreas teóricas citadas señalan una tendencia de la EF a concentrarse en la condición física, objetivista del cuerpo como máquina perfecta, y del deporte basado en la competencia y en el éxito personal e institucional. Esto trastoca las creencias de los docentes de esta asignatura, conjuntamente, con otras ideas personales y colectivas sobre el ser humano y la EF. Estas creencias afectan, profundamente, a los niños con obesidad y, doblemente, a las niñas obesas, por su condición de mujeres, de sobrepeso y de obesidad. En consecuencia, el presente estudio, cuyo eje es las creencias del personal docente de EF, hacia los niños obesos y, en particular, hacia las niñas con condición de obesidad o sin esta, resulta pertinente por su aporte sobre la importancia de la actividad y el ejercicio físico, como factor relevante en la prevención de la obesidad en niños escolares costarricenses. 


\section{Metodología}

Tipo de estudio: El artículo que se presenta es uno de los productos de una investigación "madre" desarrollada entre febrero del 2005 y setiembre 2007, la cual se ubica en el paradigma cualitativo. Se empleó el método etnográfico; en todo momento, se realizó un esfuerzo explícito para comprender los eventos de acuerdo con los significados que les confieren quienes están en un determinado medio social, en particular, evidenciar las creencias acerca de la obesidad de la docente de EF en su práctica docente.

La ubicación geográfica del centro educativo permitió realizar visitas frecuentes y en horarios amplios, de seis horas al día, de tres a cuatro veces a la semana, por aproximadamente 19 meses. Todo ello, con el fin de aportar datos significativos acerca de la enseñanza de la EF, respecto a la prevención de la obesidad en la población estudiantil de la educación general básica (EGB), para luego interpretarlos y contribuir con la comprensión de este fenómeno de estudio.

Esta investigación se enmarca también como estudio de caso, porque su propósito primordial fue comprender en profundidad las creencias de la maestra sobre la obesidad, las cuales subyacen en su enseñanza de la EF.

Por lo tanto, el estudio de casos fue adecuado para realizar un análisis intensivo y profundo de las creencias de dicha maestra, acerca del desarrollo de la obesidad en los niños de tercer año y cuarto año de la EGB, presentes en la práctica educativa de la EF. Este estudio de caso se llevó a cabo en un ambiente natural, donde se produjeron eventos y acontecimientos, los cuales fueron comprendidos, interpretados y analizados rigurosamente.

Cabe mencionar que en la investigación "madre" se predefinieron cuatro macro categorías de análisis que orientaron la recolección de los datos. En este artículo científico se presenta únicamente la información sistematizada de una macro categoría : las creencias de la docente de Educación Física sobre la obesidad de niños escolares, manifiestas en la práctica educativa y las categorías derivadas: la actividad física limitada al ámbito áulico; la obesidad, condición que dificulta la participación de los niños y niñas en EF; la exclusión de niñas y los niños con obesidad en la práctica de esta asignatura; la predilección por los estudiantes con menos dificultad; la desatención de quienes requieren más apoyo, y las creencias de la maestra modeladas por sus estudiantes.

Técnicas e instrumentos: Se utilizaron distintas técnicas de recolección de datos: la observación participante (75 crónicas de observación), entrevistas a profundidad, grupos focales y análisis de documentos.

También, se aplicó la técnica del efecto zoom, que consistió en dejar la observación general de los niños para realizar observaciones particulares de algunos con obesidad; para posteriormente retomar la observación general de los niños y las niñas de la sección. Todas las observaciones que se realizaron se anotaron a mano, en hojas en blanco y, posteriormente, se redactaron esas observaciones con formato de crónicas. 
URL: http://www.una.ac.cr/educare

CORREO: educare@una.cr

Se utilizaron varios instrumentos de distintas tecnologías con el fin de recolectar la información en la forma más fidedigna y real posible. Esto para obtener las estructuras de significados que informan y testifican los comportamientos de las personas observadas. Entre los instrumentos que se utilizaron están: la cámara fotográfica digitalizadora, para posteriormente incorporar las fotografías digitalizadas en el trabajo mediante el uso de la computadora; la grabadora de audio "tipo periodista" para grabar las entrevistas y, hojas en blanco prensadas en una tabla para registrar las notas de campo y las crónicas de observación.

Estrategias para el análisis de los datos: Se utilizó el programa ATLAS. Ti, Versión WIN: 5.0.66, al cual se asignaron 126 documentos, entre entrevistas y crónicas de observación. El programa SPSS para Windows, Versión 10.0 se utilizó para realizar algunos cálculos estadísticos de tipo descriptivos. Estos programas fueron concebidos como valiosos recursos que facilitaron la organización y la clasificación de la información, así como el tratamiento a partir de las categorías y la recuperación de textos, entre otros procedimientos analíticos que se realizaron, con el propósito de extraer los significados más relevantes sobre la obesidad de los participantes.

El modelo de análisis de datos cualitativos, propuesto por Miles y Huberman (1994), citado por Rodríguez, Gil y García (1996), también fue una estrategia que se utilizó para analizar los datos. El seguimiento de este modelo implicó realizar varias tareas que se interrelacionaron entre sí, a saber: la recolección datos, su reducción y el despliegue de los datos en matrices, y la confrontación de datos para verificar patrones, contradicciones y ausencias.

Estrategias para la validación de la información: Se utilizó la triangulación de los datos de las diferentes fuentes: observaciones, entrevistas, grupos de discusión, documentos y teoría. Así también, se realizaron devoluciones periódicas de los datos a las personas que participaron en el estudio. Estas se hicieron con el uso de la técnica de círculos de reflexión -en su mayoría se aplicó en grupo y, en algunos casos, de manera individualizada-, para que las personas participantes analizaran críticamente la información que se iba recopilando, con el propósito de verificar si la información era correcta y también de que tomaran conciencia de sus creencias y prácticas y, eventualmente, las modificaran.

Finalmente, con el objetivo de conocer la fiabilidad de la calidad científica de la categorización e interpretación de la información, se calculó "el porcentaje de acuerdo" y con base en estos cálculos, se puede afirmar que la información recabada es válida (fiabilidad del 97,0\%).

\section{Resultados}

\section{Las creencias sobre obesidad de la maestra de educación física en la práctica}

Los principales hallazgos en este estudio se orientan a la macrocategoría: Las creencias sobre obesidad de la docente en la práctica de la EF y las categorías derivadas: la actividad física limitada al ámbito áulico; la obesidad, condición que dificulta la participación de los niños y 
niñas en EF; la exclusión de las niñas y los niños con obesidad en la práctica de esta asignatura; la atención de la maestra por estudiantes con menos dificultad y la desatención de los que requieren más apoyo; las creencias de la maestra modeladas por sus estudiantes.

\section{La actividad física limitada al ámbito áulico}

En la escuela, caso de estudio, existió la creencia de que la actividad física se circunscribe al ámbito áulico. Esta creencia estuvo presente en la docente de EF y en todo el personal docente y administrativo de la escuela, y fue una limitación para que esta maestra internalizara, en forma consciente, la vinculación que existe entre la actividad física y la salud.

Esta creencia además redujo el campo de acción de la actividad física, fortaleció la visión positivista de la educación y la salud, como dos líneas paralelas que están en el mismo plano; pero que nunca se entrelazan, tal como se extrae de la siguiente crónica:

10:00 a.m. (...) En poco tiempo finalizan las clases de EF del grupo 3-2, que estoy observando donde reciben estas clases (en la plaza, frente a la escuela, caso de estudio); me acerco a la profesora de esta asignatura y le comento que después de sus clases voy a pesar y medir a las niñas y a los niños de los grupos 3-2, 4-1 y 4-3 que me faltaron de evaluar. Además, le pregunto que si le gustaría que le brinde una copia de la evaluación antropométrica de ellas y ellos. Ella me sorprende respondiéndome así: "No, no a mí no me importa que no me los dé, déselos a las maestras. Es que cómo decirte, lo mío llega hasta aquí (la profesora señala la plaza); a mí nunca me han puesto a sacar los pesos y cuánto miden; yo no tengo que ver con eso de los pesos y también yo con ellos (se refiere al personal docente) casi no tengo mucho que ver, solo una vez que el profesor (se refiere al docente de inglés) me buscó y conversamos sobre el caso (se refiere a una niña con obesidad de tercer año) y él me dijo que había hablado con la mamá de ella y con la chiquita, pero nada más".

Aquí cabe señalar, que la maestra de EF no se interesó por incentivar al alumnado a "(...) expresar una actitud de solidaridad hacia sus compañeros y compañeras, profesores y profesoras e integrantes de la comunidad en general (...)" como lo señala este fin de Los Programas de Estudio 2005 de Educación Física para I y II ciclos (MEP, 2005). No le brindó importancia al comentario que le hizo el docente de inglés sobre la problemática de una de sus alumnas con obesidad. Así también careció de una visión integral de la educación, en donde datos indicadores de la salud de los niños debieron ser conocidos por todo el personal docente para dar soluciones holísticas a problemas de salud, como la obesidad; la maestra de EF practica una forma de trabajo que desarticula las nociones de "actividad física" y "salud". 
URL: http://www.una.ac.cr/educare

CORREO: educare@una.cr

\section{La obesidad, condición que dificulta la participación de los niños y las niñas en educación física}

Para la maestra de EF, el sobrepeso y la obesidad son sinónimos, pues ambos se caracterizan por exceso de peso. Ella cree que la obesidad es una condición que puede disminuir la habilidad para moverse en la población estudiantil. Asimismo, para ella, las niñas y los niños con obesidad son lentos y con una mentalidad de negación del logro:

"J (se refiere a un niño con obesidad de tercer año) que es otro niño que ustedes observan y es una lentitud para todo; jadea al correr, perdoná la expresión, bueno al medio correr y en general no duran mucho activos. No te podría decir si es pereza o es que porque no les gusta, porque desde antes de hacer algo, yo te digo que ya ellas y ese chiquito J, se mentalizan que no lo van a lograr".

Agrega: "No te podría decir si es pereza o es que porque no les gusta".

Como se observa, los niños referidos en el testimonio, ya son etiquetados por la maestra como perdedores, pues en su creencia expresa "(...) se mentalizan que no lo van a lograr" y, como resultado, estos niños y niñas se autoexcluyen de la participación de actividades físicas, deportivas y recreativas con sus pares en la escuela, y en la comunidad.

Esta actitud de la maestra de EF, ante el desempeño de los niños obesos en la actividad física, es abordada por diferentes estudios (Moreno, 2010; Rosales et al., 2005; Toro, 2007; Vázquez, 2001) y se puede circunscribir a los docentes que creen que la finalidad de la EF es promover la condición física de los niños y la práctica del deporte, principalmente el futbol.

\section{La exclusión de niñas y niños con obesidad en la práctica de esta asignatura}

La creencia de que la lentitud es inherente a la obesidad, ya sea porque las personas con obesidad son lentas o porque "(...) en general no duran mucho activas (...)" o porque "(...) se mentalizan que no lo van a lograr(...)", provoca que la niña o el niño con obesidad no sean escogidos por sus pares para hacer equipo, pues en palabras de la maestra de EF: "(...) no sirven para competencia".

(...) a C que es otra niña, idiay bien gordita, que vos estás observando, no, no, no la escogen jaah! ¿Y por qué será que no? Porque esta chiquita no sirve para competencia y a ellos les gusta competir, ganar, son muy competitivos, verdad". 
Idiay es un patrón, los niños son más activos (...) las niñas se les pasea el alma... y es un patrón que se repite y se repite y se repite. Las niñas en general y bueno J (se refiere a un niño con obesidad de tercer año) (...) es una lentitud para todo; jadea al correr (...).

\section{Las niñas es que... bueno, hay algunas gordas o delgadas que de todos modos no} les gusta ensuciarse o sudarse o hasta despeinarse. No si vieras, vos, jugando de novelitas, etc., etc.; ellas siempre andan en otra cosa. No, si vieras que a veces con las mujeres, yo ya, como que me rindo. Ellas quieren ser delgadas pero no hacen por donde y las crespas quieren ser lacias y las muy blancas quieren estar bronceadas, pero ahí las veras sentadas y ojalá en la sombra.

En los testimonios que anteceden, la maestra anotó que los niños y las niñas con obesidad son excluidos y explicita sus creencias, en relación con los comportamientos del género femenino, pero en la práctica, no se observó que educara a sus estudiantes para conocer acerca de los comportamientos etiquetados para las niñas. Tampoco ofreció alternativas para incluir a las estudiantes en los juegos, lo que se contrapuso al fin educativo: "Propiciar en el educando y en la educanda una concepción integral de la realidad, al emplear actividades lúdicas como medio para un aprendizaje significativo y permanente" (MEP, 2005). En estos testimonios se develaron las creencias de la docente sobre el enfoque de la asignatura, mencionado anteriormente y sus creencias sobre la educación física y el género.

\section{Niñas obesas con perfil de activas, según la maestra}

Yo diría que hay más con sobrepeso que con obesidad, pero por ejemplo, K (se refiere a una niña con obesidad de cuarto año) que es una niña que es... usted la ve y es empacada, dura y además es activa. Yo si te digo esta niña, no sé cómo la han catalogado ustedes, en con sobrepeso o con obesidad, pero si esa chiquita salió con sobrepeso... esa niña no es no, porque sea sedentaria, entendéme no es que yo diga que ustedes no, o sea que ella no es gordita, yo no digo eso sino que si salió así con sobrepeso, debe ser que come mucho. $Y$ es que vos lo has visto en las clases; esta chiquita juega fútbol, la escogen los varones.

9:30 a.m. (...) Vea ahorita cómo están ellas (se refiere a dos niñas con obesidad de cuarto año) están estilando y no les importa ensuciarse. Ellas juegan con los varones, si tienen que caer al suelo, se tiran y ya (...). Yo creo que más es K que B porque K está activa siempre y B es más afeminadilla, en cambio $\mathrm{K}$ no, ella es más como natural, espontánea, quizás, no sé (...) ya te digo, las demás no, no, ellas no para nada (...)". 
URL: http://www.una.ac.cr/educare

CORREO: educare@una.cr

Llama la atención cómo la maestra expresó la creencia de que hay niñas, que independientemente de su condición de sobrepeso y de obesidad, si compartieron algunas características de los varones: "(...) esta chiquita juega fútbol, la escogen los varones". Y "Ellas juegan con los varones, si tienen que caer al suelo, se tiran y ya (...)". Las niñas en mención, son exitosas en la actividad física.

Es importante destacar que la maestra se dejó llevar por su creencia de que si las niñas asumen el rol del varón en el deporte (fuerza, competitividad, resistencia (Cremades et al., 1995) son aceptadas por los niños en el juego. Se hace presente, una vez más, que la feminidad de las niñas les impide hacer deporte.

\section{La maestra privilegia a los estudiantes con menos dificultad y desatiende a los que requieren más apoyo}

En sus clases, esta profesora "enfoca sus energías" en el alumnado que tuvo más facilidad para realizar ejercicios y desatendió a la población estudiantil que requirió de más motivación o apoyo para realizarlos. Cabe destacar, que la mayoría del alumnado que necesitó más de la atención o apoyo y motivación por parte de la maestra de EF presentó obesidad y fue de sexo femenino.

9:00 a.m., (...) La profesora se dirige al grupo: "Bien, chicos, LE es el niño que va a hacer de profesor hoy". Este niño LE presenta un estado nutricional normal. (...) C, una niña con obesidad que está en observación se acerca a la profesora y le dice: "LE nos va a matar, ya estamos cansados todos". La profesora le responde: "Usted no entendió que yo no soy hoy la profesora, dígale a LE o sino usted verá o jsiéntese ahí!" Observo que la niña no se sienta, sino que sigue en fila haciendo el calentamiento sin correr, solo camina y se nota cabizbaja. (...) "Ve lo que le dije, las niñas nada que ver y vea, ellos sí son muy competitivos, aunque están calentando, van rápido porque quieren ganar (...)".

De nuevo, en esta posición de la maestra ante la actividad física de niñas obesas mostró las creencias de la docente sobre el enfoque de la EF y de género.

9:20 a.m., (...) se dirige a un niño con estado nutricional normal y le dice: "Venga usted aquí, usted va a dirigir los ejercicios de estiramiento". Noto que MA (una niña con obesidad, caso de estudio) no realiza un estiramiento, parece que le resulta difícil, pero la profesora no la asiste. La profesora continúa diciendo que hagan grupitos de 5 filas y observo que MA se fija a ver quiénes faltan de formar grupo, pero al parecer queda solita; entonces, 
se dirige hacia la profesora para decirle que quedó sola. La profesora le contesta: "Vaya y busque a quienes les falta de rellenar el grupo y ahí se mete". Ahora observo que G (otra niña con obesidad de tercer año) dejó de hacer los ejercicios, pero la profesora no le pregunta nada y tampoco a MA (...). Observo que un niño con estado nutricional normal se sale de la cancha, donde están haciendo los ejercicios mencionados y se dirige hacia el zacate donde está la profesora de Educación Física y le dice: "Niña, MA no está haciendo nada, ya dejó de hacer los ejercicios". La profesora le contesta: "Bueno, bueno, está bien, déjela tranquila, qué importa, déjela". Luego se dirige a mí y dice: "MA es como un caso perdido, yo, yo, ya no le exijo mucho".

Este comentario, expresado por la maestra, conduce a pensar en la creencia de que no hay intervención o motivación que pueda revertir la conducta de las niñas con obesidad.

La Educación Física con una visión holística, participativa e inclusiva (Toro, 2007) tiene la finalidad de respetar las diferencias de los niños y a estimularlos a su mejoramiento integral como personas. El testimonio anterior muestra, lamentablemente, todo lo contrario: el irrespeto a la capacidad humana de mejorar su condición, pilar fundamental de la educación.

\section{Se privilegia a niños y niñas con estado nutricional normal para modelar ejercicios}

9:45 a.m. Como es costumbre, la profesora escoge a una niña y a un niño con estado nutricional normal para que dirijan los ejercicios de calentamiento (...).

De acuerdo con lo descrito, el trato que la maestra de EF les brindó a sus estudiantes con condición de obesidad produjo en ellos, y particularmente en ellas, que se desmotivaran, se rindieran, dañó su autoestima y se reprodujo el trato inequitativo y discriminatorio de la maestra, entre sus estudiantes. Esta reproducción social se da en perjuicio del desarrollo morfológico y motriz de las niñas y los niños con obesidad, y también en desmedro de la formación integral de toda la población estudiantil, uno de los pilares en los fines del Programa de Educación Física de I y II ciclos.

\section{Las creencias de la maestra las modelan sus estudiantes}

La práctica pedagógica de la maestra fue "excluidora" con los niños obesosy específicamente con las niñas que tenían esta condición; además estas conductas discriminatorias las reprodujeron sus estudiantes, cuando lideraron el grupo. Estos patrones aprendidos, mediante procesos de reproducción social, se vieron reflejados en las interacciones sociales de sus estudiantes. Por 
URL: http://www.una.ac.cr/educare

CORREO: educare@una.cr

ejemplo, en la práctica del futbol, el cual la mayoría de las niñas lo identificaron como un deporte "para varones", y donde el niño con obesidad frecuentemente cumple la función de portero, papel que él y sus pares han construido socialmente,y el que fue aceptado por la maestra.

De hecho, en las múltiples ocasiones en que se observó a los niños "organizándose para jugar un partido de fútbol", fue obvio el acuerdo tácito que existió entre ellos cuando se repartieron las funciones que cado uno desempeñó en el equipo. Sin previas expresiones verbales, la mayoría de las veces, los niños con obesidad recogieron los conos para delimitar una portería imaginaria en la plaza.

A continuación, se describen extractos de las crónicas de observación realizadas en la plaza donde los estudiantes reciben las clases de EF. Estas ejemplifican las creencias mencionadas y el comportamiento de los niños con obesidad o sin esta condición.

9:44 a.m., (...) Me acerco a J (un niño con obesidad de tercer año) para preguntarle qué va jugar en el "tiempo libre" y él me responde: "De bola, fútbol". En ese momento observo que él recoge dos conos y un compañero Ro (un niño que no presenta obesidad) le grita: "Ildiay J zoque, marque la cancha rápido!"

$J$ le responde: "¡No ve que llevo aquí los conos!"

Observo que J marca un extremo de la cancha, pone los dos conos a los lados para delimitar los límites de un arco de portería imaginario. Se ubica en medio de los dos conos. Los niños ya están jugando fútbol, la bola le llega cerca a J, pero él no corre a atajarla; se queda de pie mirando. En eso, escuho a los niños de su equipo: "iPero corra a agarrar la bola, nos van a meter el gol!"

Observo que del otro lado de la cancha, en la otra portería, está de portero un niño de estatura alta y con sobrepeso, por lo que no es caso de estudio (no presenta obesidad).

Al finalizar las clases, me acerco al niño Ro que le había gritado a J para preguntarle: "Estabas enojado con J?"

Ro contesta: "No, no, es que, solo un poco; es que yo soy el capitán del equipo y J sabe que le toca traer los conos y no le apura"

Le pregunto: "¿Por qué J sabe que le toca traer los conos, vos le habías dicho?"

Ro responde: "Es que él ya sabe y yo le recordé ahora también (...)"

Le vuelvo a preguntar: "¿Pero, por qué J sabía eso, antes de que vos le recordaras?"

Ro contesta: "Idiay porque él siempre hace de portero. (...) A J no le gusta ser otra cosa, él dice que quiere ser portero." 
Le pregunto: "¿Y el otro niño que hoy también fue portero, él sabía que le tocaba ser portero?"

Ro contesta: "Ah, usted dice MA; no es que ese no; a veces MA rota con Sa (Sa es un niño un poco menos alto que MA y con sobrepeso; por lo que no es caso de estudio).

Le pregunto: “¿Por qué ellos rotan y J no?"

Ro me responde: "Idiay porque a ellos les gusta estar en todos lados y son muy buenos metiendo goles."

Le pregunto: "¿Y J no mete goles?"

Ro me responde: "No, él no."

Luego, busco a J para conversar, pero me dice que está muy cansado, que después.

Entonces busco a la profesora de Educación Física y ella me brinda la siguiente opinión: "Sí, a J le gusta la portería y pues también seguro es que le conviene porque ya vas a ver que él no le gusta correr y vieras que sí le cuesta mucho. Yo le tengo que decir que haga "altos" para que respire. Además, los chicos son muy competitivos y no les gusta que J no corra a patear la bola; idiay, entonces lo ponen de portero (...) El año pasado, un chiquito que no está ya aquí en esta escuela, le dijo a J que él tenía que ser portero porque tapaaaaba toooodo, verdad. Entonces, yo inmediatamente, porque J siempre ha sido así, como vos lo estás viendo ahora; entonces, le dije a la maestra que le mandara un recado a la máma de ese niño sobre eso y, entonces, ninguno, no lo han vuelto a molestar porque saben que yo no lo permito".

Es interesante observar cómo en esta situación media la creencia de que "el boleteo" de la maestra ordinaria tiene el poder de corregir la "chota" del estudiante hacia el niño obeso. La docente de EF no se apropió de su misión formadora para reflexionar con sus estudiantes sobre estos comportamientos de violencia verbal hacia los niños obesos.

9:50 a.m., (...) Cuando terminaron de jugar fútbol me acerqué y les pregunté: ¿Cuáles son las cualidades para ser portero?

$P$ (niño con obesidad y activo) responde: "A mí me gusta mucho y como ellos dicen que yo soy alto".

Ti (niño que no presenta obesidad y considerado "educado" por la profesora de Educación Física, porque está en una Escuela de fútbol) responde: "Yo puedo jugar de todo". 
URL: http://www.una.ac.cr/educare

CORREO: educare@una.cr

Entonces le pregunto: “Quién no podría jugar de todo?"

Ti dice: "Idiay no sé, tal vez alguno que no tenga aire para correr, ehh, puede ser que la "Gorda bella" (risas)."

P: "Sí es que vea, es mejor que $\mathbf{E}$ (un niño con obesidad que le dicen la "Gorda bella") sea portero porque él no corre muchísimo y hoy no quiso; entonces como Ti también sabe atrapar bien y de todo, entonces Ti hizo de portero hoy".

Busco a E para conocer su opinión y me responde:

E: "Yo soy el portero oficial (risas), porque soy bueno atrapando y yo, a veces puedo correr rápido todo el partido, pero a veces no porque me canso, y porque un día me doblé un tobillo (...). Hoy no quise ser portero porque LE (un niño líder que no presenta obesidad) es un mandón, fue solo por llevarle la contraria, porque a veces juega de vivo.".

Le pregunto: ¿Y no te gustaría jugar en otro puesto?

E responde: "Mmm, como hoy digamos (...), mmm, no sé".

Se observa en este testimonio cómo las creencias de la docente sobre la actividad física y el género las reproducen los estudiantes, llaman "Gorda bella" a un niño que por su condición de obesidad es lento y poco competitivo, rasgos que los resultados de investigación dan al género femenino (Cremades et al., 1995; Nuñez, 2007). Parece que un varón con estas características se asocia con los comportamientos de las niñas, explicitadas por la maestra así: "(...) esta chiquita (refiriéndose a una niña obesa) no sirve para competencia y a ellos les gusta competir".

De lo expuesto, los niños y las niñas con obesidad han descodificado los mensajes implícitos, emitidos por sus pares y la maestra de EF: "saben que les toca hacer de porteros", "porque ellos son lentos", "no duran mucho activos", "se mentalizan que no lo van a lograr" y "no sirven para competencia". Esta descodificación niega los fines de la EF, en cuanto a fortalecer las destrezas de los niños y niñas con los ejercicios físicos, los deportes y la creatividad, para la consecución de una vida saludable y una convivencia pacífica y solidaria entre los seres humanos con condición de obesidad o sin ella.

\section{Discusión}

Se puede afirmar que, en el caso en estudio, se identifica la visión positivista de la educación y de la salud en la práctica pedagógica de la maestra de EF, porque su interés está en el conocimiento técnico (Giroux, 1997; Rosales et al., 2005), en su medición y cuantificación del logro, sin estimar las limitaciones de algunos niños y niñas para obtenerlo. Hay ausencia de valoración de procesos de adecuación para estudiantes con condición de obesidad y de otras enfermedades, cuya participación debe ser orientada por criterios flexibles y adecuados a sus necesidades. 
El conocimiento es evaluado mediante el uso de métodos analíticos hipotético-deductivos o empíricos. Estos clasifican, encasillan y, sobre todo, controlan al estudiantado. Con frecuencia la maestra empleó la represión y el castigo para controlar la conducta de las niñas y los niños, a saber:

"(...) tiene que quedarse haciendo algo de algún deporte y que lo exponga la próxima vez, porque sino de dónde cojo la nota; yo tengo que evaluarlo".

Este testimonio se refiere a un niño que no participa en futbol y ejemplifica una conceptualización del sustrato técnico-instrumental "(...) lógica que desatiende los procesos y privilegia los resultados medibles, transformando la evaluación en un proceso parcial e insuficiente, dado que se parte del supuesto de que todo lo que se enseña se aprende (...)" (Prieto, 2008, p. 126).

Por lo tanto, la evaluación del estudiante en mención se circunscribe a una medida de control "de dónde cojo la nota"; hay que poner al estudiante a hacer algo para tener una nota, sin tomar en cuenta si hubo o no aprendizaje, ni por qué este niño no practica el deporte. La evaluación se convierte en trámite más que en un proceso de aprendizaje.

Siguiendo a Moreno (2010), Rosales et al. (2005) y Vázquez (2001), la práctica educativa de la docente del presente estudio se ubica en los enfoques cuyos ejes son la condición física y el deporte. En esta visión tienen privilegio los niños con condición de peso normal, que pueden realizar el ejercicio, la actividad física o el deporte con agilidad y sin agotarse. En este sentido, los niños obesos constituyen un problema para la EF y, principalmente, para el deporte del fútbol, porque son poco competitivos, se cansan muy rápido y no son aceptados por los niños con peso normal, dado que a estos le gusta ganar los partidos. Las niñas participantes en el estudio son las menos favorecidas, ya que por ser mujeres, según la docente se caracterizan por ser pasivas, con actitudes negativas hacia la participación en el deporte; esta creencia de la maestra se valida con los resultados de la investigación de Cremades et al. (1995), los cuales identifican a las niñas con actividades culturales-artísticas, manuales y prácticas sociales y no con aquellas dedicadas a actividades físico-recreativas y lúdicas, las cuales adjudican a los niños .

En las niñas, las creencias, además de las limitaciones que les otorgan por su condición de género en la práctica de la EF, se suman a la condición de obesidad; son, entonces, etiquetadas en su condición física como perezosas, lentas, indiferentes hacia la actividad física; y en su comportamiento social como delicadas "no les gusta ensuciarse", "creídas", "vanidosas" (Núñez-Rivas, 2007).

Es importante destacar que muchas veces las creencias sobre el ejercicio y la actividad física, así como sobre el deporte en relación con el género y la obesidad, no son de naturaleza genética o biológica, sino psicosocial, y afectan la baja autoestima y la poca aceptación de la imagen corporal en los sujetos. Estas creencias deben conocerse e intervenir aquellas prácticas 
URL: http://www.una.ac.cr/educare

CORREO: educare@una.cr

educativas cuyo enfoque no sea inclusivo, porque estas son decisivas para que los niños y las niñas con obesidad tengan interés en su salud; se sientan comprometidos con el cuidado de sus propios cuerpos, y sean capaces de llevar a cabo prácticas de vida consecuentes con una adecuada salud corporal (Melnyk et al., 2006).

Desde la perspectiva teórica, los "Programas de Estudio de Educación Física para I y II ciclos", del Ministerio de Educación Pública (MEP) (2005) explicitan creencias sobre una población estudiantil que debe desarrollarse reconociendo que las actividades físicas, deportivas y recreativas son medios para gozar de una adecuada condición física, adquirir destrezas y habilidades psicomotrices. Estas intenciones se enmarcan en una concepción con énfasis en la condición física, de carácter más terapéutico que psicosocial, en donde la atención a la salud, en este caso la obesidad, se atiende como problema de salud dado y no como prevención de la enfermedad. Por lo tanto, el enfoque biologista del programa de EF encierra las creencias de que la obesidad es consecuencia de la genética o de condición física, en desmedro del concepto de obesidad, polícromo, estudiado desde diferentes aristas: biológicas, psicosociales, culturales, en las que se trata la obesidad como una enfermedad de carácter multifactorial. Desde esta perspectiva, un enfoque de la EF holístico, participativo, inclusivo, como el que presenta Toro (2007), es el adecuado en los programas de EF en el ciclo básico costarricense.

Las creencias de la maestra de EF, reflejadas en la práctica docente, no son congruentes con las intenciones expresadas en el programa de esta asignatura (documento escrito). Si bien, el programa se centra en un enfoque que tiene como pilar la condición física y recreativa de los niños, la práctica de la maestra se basa en creencias sobre la condición física de niños y niñas distorsionadas, por su condición de género y de obesidad, que no les permite desarrollarse físicamente, disfrutar de la práctica del deporte, particularmente del futbol, y les afecta la autoestima por la discriminación de los pares con peso normal.

Al respecto, Chambliss, Finley y Blair (2004) hicieron un estudio para evaluar las actitudes y las creencias de estudiantes de la carrera de Ciencias del Ejercicio (en Costa Rica, esta carrera se podría homologar con Educación Física). En las respuestas a pruebas construidas para valorar estos aspectos encontraron actitudes y creencias "negativas" hacia las personas con obesidad.

Entre los hallazgos del estudio en mención, coincidentes con lo encontrado en la presente investigación, están los estereotipos de que las personas con obesidad son perezosas. Asimismo, reportaron que las personas con obesidad de sexo femenino, caucásicas y que vivían en zonas poco pobladas fueron asociadas $(P<0.0001)$ con creencias y actitudes negativas.

El resultado del presente estudio con respecto a la selección de las niñas y niños con peso normal, por parte de la maestra, para que modelaran ejercicios y jugaran futbol, se pueden analizar a la luz de una "cultura del consumo". Aquí en la escuela, el estudiante pareciera que debe adecuarse al "nuevo ciudadano-consumidor" y a la "nueva ciudadana-consumidora". Pues así lo muestra la práctica pedagógica de la maestra de EF, al seleccionar cuerpos de estudiantes, con características para la competencia y el modelaje. 
Asimismo, de acuerdo con las creencias de la maestra de EF, los niños son más activos que la niñas en general, sean estas "gordas o delgadas", pues ella cree que las niñas no realizan ejercicios porque "no les gusta ensuciarse o sudarse o hasta despeinarse", lo que podría asociarse a los modelos de mujeres proyectados por las "barbies", como producto del consumo.

La situación descrita es preocupante y debe ser socializada por el personal docente de $\mathrm{EF}$, porque en su mayoría no son conscientes de las creencias que alimentan su práctica y están obstaculizando el desarrollo pleno del ser humano: el biológico, físico, emocional, social y espiritual del estudiantado en edad escolar. ¿Cuál ser humano contestaría a la pregunta si es importante ser aceptado en forma negativa? Y, tristemente, observamos que el maestro en general y la maestra del estudio, quienes tienen creencias de cultura, de corte neoliberal, forjada en el éxito y la competencia, lastiman una de las necesidades más hondas del corazón humano: la de ser apreciado (Van Breemen, 1974). De ahí la importancia de comprender el fenómeno de las creencias en nuestras vidas y, primordialmente, en la práctica de los maestros y maestras, y de los padres y madres de familia, quienes forman una niñez que desea la aceptación de los demás, porque un niño aceptado es un niño feliz.

\section{Referencias}

Allison, P. C., Pissanos, B. W., Turner, A. P. y Law, D. R. (2000). Preservice Physical Educators' Epistemologies of Skilfulness [Las epistemologías sobre las destrezas de los educadores físicos en servicio]. Journal of Teaching in Physical Education, 19(2), 141-161.

Bernard, M. (1985). El cuerpo. España: Paidós.

Chambliss H. O., Finley, C. E. y Blair S. N. (marzo, 2004). Attitudes Toward Obese Individuals Among Exercise Science Students [Las Actitudes hacia las personas obesas entre los estudiantes de ciencias del ejercicio]. Medicine and Science in Sports and Exercice,36(3), 468-474.

Council on Sports Medicine and Fitness; Council on School Health. (2006). Active Healthy Living: Prevention of Childhood Obesity Through Increased Physical Activity [Vida saludable y activa: La prevención de la obesidad infantil mediante el incremento de la actividad física]. Pediatrics, 117(5), 1834-1842. doi: 10.1542/peds.2006-0472

Cremades, M. Á, Álvarez, C., Urruzola, M. J., Jaramillo, C. y Hernández, G. (1995). Coeducación y tiempo libre. Madrid: Popular.

Davis, B. y Carpenter, C. (marzo, 2009). Proximity of Fast-Food Restaurants to Schools and Adolescent Obesity. [Proximidad de restaurantes de comida rápida a las escuelas y la obesidad en adolescentes]. American Journal of Public Health, 99(3), 505-510.

DeMattia, L, Lemont L. y Meurer, L. (enero, 2007). Do Interventions to Limit Sedentary Behaviours Change Behaviour and Reduce Childhood Obesity? A Critical Review of the Literature [¿Las intervenciones que limitan el comportamiento sedentario cambian comportamientos y 
URL: http://www.una.ac.cr/educare

CORREO: educare@una.cr

reducen la obesidad infantil?]. Obesity Reviews, 8(1), 69-81. Recuperado de http://www. ncbi.nlm.nih.gov/pubmed?Db=pubmed\&Cmd=ShowDetailView\&TermToSearch=1721279 7\&ordinalpos=2\&itool=EntrezSystem2.PEntrez.Pubmed.Pubmed_ResultsPanel.Pubmed_ RVDocSum

Denis, D. (1980). El cuerpo enseñado. Barcelona: Paidos.

Díaz, L. E. (28 de octubre de 2012). Ticos pierden la cintura por sus malos hábitos alimentarios. La Nación (El país). Recuperado de http://www.nacion.com/2012-10-28/EIPais/ticos-pierdenla-cintura-por-sus-malos-habitos-alimentarios.aspx

Durán, E. (2001). Las creencias de los profesores: Un campo para deliberar en los procesos de formación. Revista de Acción Educativa, 1(1).

Espinal, A. (2006). La sociología del cuerpo. Psiopedagogía.com. Recuperado de http://www. psicopedagogia.com/sociologia-cuerpo

Floriani, V., Kennedy, C. (febrero, 2007). Promotion of Physical Activity in Primary Care for Obesity Treatment/Prevention in Children [Promoción de la actividad física en la atención primaria para el tratamiento de la obesidad/prevención en niños]. Current Opinion in Pediatrics, 19(1), 99-103. Recuperado de http://www.ncbi.nlm.nih.gov/pubmed/17224670

Giroux, H. (1997). Teoría y resistencia en educación: Una pedagogía para la oposición. México: Siglo $\mathrm{XXI}$.

Greenleaf, C. y Weiller, K. (2005). Perceptions of Youth Obesity Among Physical Educators [Percepciones acerca de los jóvenes obesos entre los docentes de la educación física]. Social Psychology of Education, 8(4), 407-423. doi: 10.1007/s11218-005-0662-9

Habermas, J. (1999). Teoría de la acción comunicativa. Madrid: Taurus.

Hernández, J. L. (1996). La construcción histórica y social de la educación física: El currículo de la LOGSE, ¿una nueva definición de la educación física escolar? Revista de Educación, 311, 51-76.

Kaur, H, Hyder, M. L. y Poston, W. S. (2003). Childhood Overweight: An Expanding Problem [EI sobrepeso infantil: Un problema en expansión]. Treatments in Endocrinologic, 2(6), 375-388. Recuperado de http://www.ncbi.nlm.nih.gov/pubmed/15981942

Melnyk, B. M., Small, L., Morrison-Beedy, D., Strasser, A., Spath, L., Kreipe, R., Blankenstein, S. (noviembre-diciembre, 2006). Mental Health Correlates of Healthy Lifestyle Attitudes, Beliefs, Choices, and Behaviors in Overweight Adolescents [La salud mental y su correlación con actitudes hacia la vida sana, creencias, decisiones y comportamientos en adolescentes con sobrepeso]. Journal of Pediatr Health Care, 20(6), 401-406. 
Ministerio de Educación Pública (MEP). (2005). Programa de Educación Física. San José, Costa Rica: Autor.

Moreno, A. (2010). Percepciones del profesorado universitario de educación física en relación a la función de transformación social de la educación física escolar: El caso de Chile (Tesis doctoral). Granada: Universidad de Granada. Recuperado de http://0-hera.ugr.es.adrastea.ugr.es/ tesisugr/19580046.pdf

Morin, E. (2003). El método. La humanidad de la humanidad. La identidad humana. Madrid: Cátedra.

Núñez-Rivas, H. P. (2007). Las creencias sobre obesidad de estudiantes de la educación general básica. Revista Educación, 31(1),145-164.

Núñez-Rivas, H. P., Monge-Rojas, R., León, H. y Roselló, M.s (enero, 2003). Prevalence of Overweight and Obesity Among Costa Rican Elementary School Children [Prevalencia de sobrepeso y obesidad entre los niños costarricenses en edad escolar]. Revista Panamericana de Salud Pública, 13(1), 24-32.

Portela, H. (noviembre, 2001). Paradigmas del cuerpo en la educación física. Revista Digital Buenos Aires, 7(42). Recuperado de http://www.efdeportes.com/efd42/paradig.htm

Pozo, J. I. (2000). Concepciones de aprendizaje y cambio educativo. Ensayos y Experiencias, 6(33), 4-13.

Prieto, M. (enero-junio, 2008). Creencias de los profesores sobre evaluación y efectos indicentales. Revista de Pedagogía, 29(84), 123-144.

Rashmi and Sushma Jaswal. (2012). Beliefs About Obesity Among Parents, Peers and Teachers of Adolescents from Punjab, India [Creencias acerca de la obesidad entre progenitores, pares y maestros de adolescentes provenientes de Punjab, India]. Ethno Med, 6(1): 39-43. Recuperado de http://www.krepublishers.com/02-Journals/JP/JP-02-0-000-11-Web/JP-021-000-11-PDF/JP-02-1-045-11-044-Rashmi/JP-02-1-045-11-044-Rashmi-Tt.pdf

Rennie, K. L., Wells, J. C., McCaffrey, T. A. y Livingstone, M. B. (2006). The Effect of Physical Activity on Body Fatness in Children and Adolescents [El efecto de la actividad física sobre la grasa corporal de niños y adolescentes]. Proceedings of the Nutrition Society, 65(4),393-402. doi: 10.1079/PNS2006515

Rodríguez, G., Gil, J. y García E. (1996). Metodología de la investigación cualitativa. Granada: Ediciones Ajibe.

Rosales, F. R., Vicente, V. y Fleitas, R. (marzo, 2005). Creencias de resultados, expectativas y motivos del profesorado de la educación física: Su influencia en el desarrollo del proceso docente educativo. Revista Digital Buenos Aires, 10(82). Recuperado de http://www.efdeportes.com/ efd82/pef.htm 
URL: http://www.una.ac.cr/educare

CORREO: educare@una.cr

Singh, A. S, Chin A Paw, M. J., Kremers, S. P., Visscher, T. L., Brug., J y van Mechelen, W. (diciembre, 2006). Design of the Dutch Obesity Intervention in Teenagers (NRG-DOiT): Systematic Development, Implementation and Evaluation of a School-Based Intervention Aimed at the Prevention of Excessive Weight Gain in Adolescents [Diseño de una intervención holandesa en adolescentes (NRG-DOiT): Desarrollo sistemático, implementación y evaluación de una intervención basada en la escuela dirigida a la prevención del aumento excesivo de peso en adolescentes]. BMC Public Health, 6(304), 1-15. doi: 10.1186/1471-2458-6-304 YA

Stice E., Shaw, H., Marti, C. N. (setiembre, 2006). A meta-analytic review of obesity prevention programs for children and adolescents: the skinny on interventions that work [Una revisión meta-analítica de los programas para niños y adolescentes: Cómo funciona el concepto de delgado en las intervenciones]. Psychological Bull, 132(5), 667-691. doi: 10.1037/00332909.132.5.667.

Story, M, Neumark-Sztainer, D. y French, S. (marzo, 2002). Individual and Environmental Influences on Adolescent Eating Behaviors [Influencias individuales y ambientales sobre las conductas alimentarias de los adolescentes]. Journal of the American Dietetic Association, 102(3), S40-S51.

Toro, S. (2007). Una aproximación epistemológica a la didáctica de la motricidad desde el discurso y práctica docente. Estudios Pedagógicos, 33(1), 29-43. Recuperado http://www.redalyc.org/articulo.oa?id=173514133002

Trueba, S. (enero, 2008). Concepciones de género en las clases de educación física. Revista Digital Buenos Aires, 12(116). Recuperado de http://www.efdeportes.com/efd116/concepcionesde-genero-en-las-clases-de-educacion-fisica.htm

Van Breemen, P. G. (1974). El coraje de aceptar la aceptación. Denville, Nueva Jersey: Dimension Brooks.

Vázquez, B. (2001). Los fundamentos de la educación física. En B. Vázquez (Coord.). Bases educativas de la actividad física y el deporte (pp. 47-68). Madrid: Editorial Síntesis.

\section{Cómo citar este artículo en APA:}

Núñez-Rivas, H. P., Campos-Saborío, N., Holst-Schumacher, I. y Alfaro-Mora, F. V. (2013). Las creencias de la docente de educación física sobre la obesidad en la niñez de edad escolar. Revista Electrónica Educare, 17(2), 5-30. Recuperado de http://www.revistas. una.ac.cr/index.php/EDUCARE/issue/current

Nota: Para citar este artículo en otros sistemas puede consultar el hipervínculo "Como citar el artículo" en la barra derecha de nuestro sitio web:

http://www.revistas.una.ac.cr/index.php/EDUCARE/index 